\title{
VARIANCE OF THE ISOTROPIC UNIFORM SYSTEMATIC SAMPLING
}

\author{
JIŘÍ JANÁČEK ${ }^{1}$ AND DANIEL JIRÁK ${ }^{2,3}$
}

${ }^{1}$ Department of Biomathematics, Institute of Physiology of the Czech Academy of Sciences, Vídeňská 1083, 14220 Prague, Czech Republic, ${ }^{2}$ MR Unit, Department of Diagnostic and Interventional Radiology, Institute for Clinical and Experimental Medicine, Vídeňská 1958/9, 14021 Prague, Czech Republic, ${ }^{3}$ Institute of Biophysics and Informatics, First Faculty of Medicine, Charles University, Prague, Czech Republic

e-mail: jiri.janacek@fgu.cas.cz, daniel.jirak@ikem.cz

(Received July 15, 2019; revised November 8, 2019; accepted December 6, 2019)

\begin{abstract}
The integral of a smooth function with bounded support over a set with finite perimeter in Euclidean space $\mathbb{R}^{d}$ is estimated using a periodic grid in an isotropic uniform random position. Extension term in the estimator variance is proportional to the integral of the squared modulus of the function over the object boundary and to the grid scaling factor raised to the power of $d+1$. Our result generalizes the Kendall-Hlawka-Matheron formula for the variance of the isotropic uniform systematic estimator of volume.
\end{abstract}

Keywords: isotropic design, spatial statistic, stereology, systematic samplings, variance.

\section{INTRODUCTION}

Estimation in spatial statistics and stereology is based on geometric sampling of spatial variables. Systematic sampling is preferable to simple random sampling for its efficiency. According to the KendallHlawka-Matheron formula (Kendall, 1948; Hlawka, 1950; Matheron, 1965), the extension term in an asymptotic expansion of the variance of a volume estimate using a point grid at an isotropic uniform random (IUR) position with respect to the grid scaling factor is proportional to the surface area of the object and to the power function of the grid scaling factor. The formula is used in the case of specific volume measurements (Gundersen and Jensen, 1987) and evaluates the mean variance of the volume estimate of a random set (Kieu and Mora, 2004). The formula can be generalised to isotropic sampling using an arbitrary regular grid (Janáček, 2006; Kieu and Mora, 2006). The grid is, mathematically speaking, a periodic Borel $\sigma$-finite measure, usually the Hausdorff measure, e.g. the counting measure on the points or $\mathscr{H}^{1}$ on the lines.

We examined the variance of the estimator for the integral of a function $f$ over $K \subset \mathbb{R}^{d}$,

$$
\int_{K} f(x) d x,
$$

obtained by replacing the ordinary Lebesque measure by a randomly rotated and shifted periodic Borel $\sigma$ finite measure.

We prove the generalised Kendall-HlawkaMatheron formula for variance of an integral estimator using a grid in isotropic uniform random position provided that $K$ has finite perimeter and $f$ is a smooth function with bounded support in Theorem 4. The asymptotics in the generalised formula is established using harmonic analysis and second Wiener's Tauberian theorem in Theorem 1, whose proof is from (Wiener, 1933, Theorem 21) modified to isotropic situation in arbitrary dimension as in (Janáček, 2008, Lemma 2.5). The constant in the asymptotics expansion is obtained by the theory of functions with bounded variation (Ambrosio, 2000) in Theorem 3 in a similar way as in (Galerne, 2011, Theorem 14).

\section{VARIANCE OF THE ESTIMATOR}

Periodicity in the Euclidean space $\mathbb{R}^{d}$ is defined based on periods being elements of the point lattice $\mathbf{T}=A \mathbb{Z}^{d}$, where $\mathbb{Z}^{d}$ are the points with integral coordinates and $A \in \mathbb{R}^{d \times d}$ is a regular matrix. We define the fundamental region of $\mathbf{T}$ as $F_{\mathbf{T}}=A[0,1)^{d}$, its volume being $\operatorname{det} A$. The spatial intensity of $\mathbf{T}$ is $\alpha=(\operatorname{det} A)^{-1}$. The lattice dual to $\mathbf{T}$ is $\mathbf{T}^{*}=A^{-1} \mathbb{Z}^{d}$.

The $\sigma$-finite measure $\mu$ on $\mathbb{R}^{d}$ is a $\mathbf{T}$-periodic measure if $\mu(K+x)=\mu(K)$ for all $x \in \mathbf{T}$ and any Borel set $K$. Let $\lambda$ be the intensity of $\mu$ equal to $\alpha \mu\left(F_{\mathbf{T}}\right)$.

The IUR position of the grid is parametrized by the fundamental region of the lattice of periods and by the rotations. Application of $d$-dimensional harmonic analysis yields the asymptotics of the variance presented in Theorem 1. The basic notions and properties concerning the Fourier transform and 
convolution of functions are summarized as follows and can be found in Bochner (1949).

For $f \in \mathbf{L}^{1}\left(\mathbb{R}^{d}\right)$ the Fourier transform $\mathscr{F} f$ is the function of frequency $\xi$

$$
\mathscr{F} f(\xi)=\int_{\mathbb{R}^{d}} f(x) \exp (-2 \pi i x \xi) d x .
$$

The coefficient $-2 \pi i$ is replaced by $2 \pi i$ in the inverse Fourier transform.

For reflection $\widehat{f}(x)=f(-x)$, the Fourier transform is the complex conjugate $\mathscr{F} \widehat{f}=\overline{\mathscr{F} f}$. by

The convolution of $f_{1}$ and $f_{2} \in \mathbf{L}^{1}\left(\mathbb{R}^{d}\right)$ is defined

$$
f_{1} \star f_{2}(x)=\int_{\mathbb{R}^{d}} f_{1}(x-y) f_{2}(y) d y .
$$

We have $\mathscr{F}\left(f_{1} \star f_{2}\right)=\mathscr{F} f_{1} \mathscr{F} f_{2}$ by convolution theorem for Fourier transform.

The rotation $M \in \mathbf{S O}_{d}$ (special orthogonal group) of a function $f$ is defined as

$$
M f(x)=f\left(M^{-1} x\right) .
$$

The Fourier transform of a spherically symmetric function $f$ (i.e. $M f=f$ for any $M$ ) is spherically symmetric. Using the same notation for a spherically symmetric function and its radial function $(f(x)=$ $f(\|x\|)$ and $\mathscr{F} f(\xi)=\mathscr{F} f(\|\xi\|))$, we have $r^{d-1} f(r) \in \mathbf{L}^{1}\left(\mathbb{R}^{+}\right)$and the radial function of $\mathscr{F} f$ is the Hankel transform of the radial function of $f$ :

$$
\mathscr{F} f(\rho)=2 \pi \rho^{1-\frac{d}{2}} \int_{0}^{\infty} r^{\frac{d}{2}} J_{\frac{d}{2}-1}(2 \pi \rho r) f(r) d r,
$$

where $J_{\frac{d}{2}-1}$ is Bessel function of first kind.

For $f \in \mathbf{L}^{1}\left(\mathbb{R}^{d}\right) \cap \mathbf{L}^{2}\left(\mathbb{R}^{d}\right)$ the covariogram $g_{f}$ is the function

$$
g_{f}(x)=\int_{\mathbb{R}^{d}} f(y+x) \overline{f(y)} d y .
$$

Obviously $g_{f}(-x)=\overline{g_{f}(x)}$. The Parseval theorem and the convolution theorem yield $\mathscr{F} g_{f}=|\mathscr{F} f|^{2} \in$ $\mathbf{L}^{1}\left(\mathbb{R}^{d}\right)$ and $g_{f}=\mathscr{F}^{-1} \mathscr{F} g_{f}$ by the Fourier inversion theorem.

The isotropic covariogram $\mathfrak{g}_{f}$ of $f$ is the real function

$$
\mathfrak{g}_{f}(\|x\|)=\int_{\mathbf{S O}_{d}} g_{M f}(x) d p(M),
$$

where $p(M)$ is the invariant probabilistic measure on $\mathbf{S O}_{d}$. Then

$$
\begin{gathered}
\mathscr{F} \mathfrak{g}_{f}(\|\xi\|)=\int_{\mathbf{S O}_{d}} \mathscr{F} g_{M f}(\xi) d p(M)= \\
=\int_{\mathbf{S O}_{d}}|\mathscr{F} M f|^{2}(\xi) d p(M) .
\end{gathered}
$$

$\mathscr{F} \mathfrak{g}_{f}$ is nonegative, $\rho^{d-1} \mathscr{F} \mathfrak{g}_{f}(\rho) \in \mathbf{L}^{1}\left(\mathbb{R}^{+}\right)$and

$$
\mathscr{F} \mathscr{F} \mathfrak{g}_{f}=\mathfrak{g}_{f} .
$$

Harmonic analysis of periodic measures leads to Fourier series. The Fourier coefficient of a $\mathbf{T}$-periodic $\sigma$-finite Borel measure $\mu$ with index $\xi \in \mathbf{T}^{*}$ is

$$
m_{\xi}=\alpha \int_{F_{\mathbf{T}}} \exp (-2 \pi i x \xi) d \mu(x) .
$$

The convolution of a $\sigma$-finite Borel measure $\mu$ in $\mathbb{R}^{d}$ with a function $f \in \mathbf{L}^{1}\left(\mathbb{R}^{d}\right)$ is

$$
f \star \mu(x)=\int_{\mathbb{R}^{d}} f(x-y) d \mu(y) .
$$

Let $\mu$ be a T-periodic measure and let $f \in$ $\mathbf{L}^{1}\left(\mathbb{R}^{d}\right) \cap \mathbf{L}^{2}\left(\mathbb{R}^{d}\right)$. Then the convolution is a periodic function and its Fourier series in the space $\mathbf{L}^{2}\left(F_{\mathbf{T}}, \alpha \lambda^{d}\right)$ is

$$
f \star \mu(x)=\sum_{\xi \in \mathbf{T}^{*}} m_{\xi} \mathscr{F} f(\xi) \exp (2 \pi i x \xi) .
$$

Let $J_{f}=\int_{\mathbb{R}^{d}} f(y) d y$. Then the mean and variance of $f \star \mu$ are

$$
\begin{gathered}
\alpha \int_{F_{\mathbf{T}}} f \star \mu(x) d x=m_{0} \mathscr{F} f(0)= \\
=\lambda \int_{\mathbb{R}^{d}} f(x) d x=\lambda J_{f}
\end{gathered}
$$

and

$$
\begin{aligned}
& \alpha \int_{F_{\mathbf{T}}}\left|f \star \mu(x)-\lambda J_{f}\right|^{2} d x= \\
& \quad=\sum_{\xi \in \mathbf{T}^{*}}\left|m_{\xi}\right|^{2}|\mathscr{F} f(\xi)|^{2},
\end{aligned}
$$

respectively. Eq. 4 follows from the Plancherel theorem for Fourier series in Hilbert space (Rudin, 1987, Theorem 4.18).

Let $\mu$ be a T-periodic measure and $u \in \mathbb{R}^{+}$. We define the $u \mathbf{T}$-periodic measure $\mu^{u}$ by the formula $\mu^{u}(K)=u^{d} \mu\left(u^{-1} K\right)$. The Fourier coefficient of $\mu^{u}$ with index $u^{-1} \xi, \xi \in \mathbf{T}^{*}$, is $m_{\xi}$. 
We define the coefficient $C_{\mu}$ of a T-periodic measure $\mu$ as

$$
C_{\mu}=\frac{1}{2 \pi^{2} d \kappa_{d}} \sum_{\xi \in \mathbf{T}^{*}}^{\xi \neq 0} \frac{\left|m_{\xi}\right|^{2}}{\|\xi\|^{d+1}},
$$

where $\kappa_{d}=\pi^{\frac{d}{2}} \Gamma\left(\frac{d}{2}+1\right)^{-1}$ is the volume of the unit ball in $\mathbb{R}^{d}$.

Theorem 1. Let $f \in \mathbf{L}^{1}\left(\mathbb{R}^{d}\right) \cap \mathbf{L}^{2}\left(\mathbb{R}^{d}\right)$ and let $\mathfrak{g}_{f}^{\prime}(0+)$, the right derivative of its isotropic covariogram in 0 , exists and is different from 0 , let $\mu$ be a $\mathbf{T}$-periodic measure and $u>0$. Then the variance of $\widehat{M f} \star \mu^{u}(x)$, where $x$ and $M$ are uniform random, is

$$
\begin{gathered}
\int_{\mathbf{S O}_{d}} \alpha \int_{F_{\mathbf{T}}}\left|\widehat{M f} \star \mu^{u}(x)-\lambda J_{f}\right|^{2} d x d p(M)= \\
=C_{\mu}\left(-\frac{d \kappa_{d}}{\kappa_{d-1}} \mathfrak{g}_{f}^{\prime}(0+)\right) \Phi_{f}\left(u^{-1}\right) u^{d+1},
\end{gathered}
$$

where $J_{f}=\int_{\mathbb{R}^{d}} f(y) d y$ and $\Phi_{f}$ is a nonnegative function on $\mathbb{R}^{+}$such that $\lim _{x \rightarrow+\infty} \frac{1}{x} \int_{0}^{x} \Phi_{f}(y) d y=1$.

Proof. The mean value of $\widehat{M f} \star \mu^{u}(x)$ is $\lambda \int_{\mathbb{R}^{d}} f(y) d y=\lambda J_{f}$ for any $M \in \mathbf{S O}_{d}$ by Eq. 3 . The average of Eq. 4 with respect to rotations is

$$
\begin{gathered}
\underset{\mathbf{S O}_{d}}{\int \alpha} \int_{F_{\mathbf{T}}}\left|\widehat{M f} \star \mu^{u}(x)-\lambda J_{f}\right|^{2} d x d p(M)= \\
=\sum_{\xi \in \mathbf{T}^{*}}^{\xi \neq 0}\left|m_{\xi}\right|^{2} \mathscr{F} \mathfrak{g}_{f}\left(u^{-1}\|\xi\|\right) .
\end{gathered}
$$

We set

$$
\begin{gathered}
\Psi_{f}(y)=-\frac{2 \pi^{2} \kappa_{d-1} y^{d+1}}{\mathfrak{g}_{f}^{\prime}(0+)} \mathscr{F} \mathfrak{g}_{f}(y), \\
\Phi_{f}(x)=\sigma_{\mathbf{T}}^{-1} \sum_{\xi \in \mathbf{T}^{*}}^{\xi \neq 0} c_{\xi} \Psi_{f}(\|\xi\| x), \\
c_{\xi}=\left|m_{\xi}\right|^{2}\|\xi\|^{-d-1}, \sigma_{\mathbf{T}}=\sum_{\xi \in \mathbf{T}^{*}}^{\xi \neq 0} c_{\xi}, \\
L(u)=\frac{1}{2 \pi^{2} \kappa_{d-1}}\left(d \kappa_{d}-2 \pi u^{1-\frac{d}{2}} J_{\frac{d}{2}-1}(2 \pi u)\right) .
\end{gathered}
$$

Then the variance is

$$
\sum_{\xi \in \mathbf{T}^{*}}^{\xi \neq 0}\left|m_{\xi}\right|^{2} \mathscr{F} \mathfrak{g}_{f}\left(u^{-1}\|\xi\|\right)=
$$

$$
\begin{gathered}
=\frac{-\mathfrak{g}_{f}^{\prime}(0+)}{2 \pi^{2} \kappa_{d-1}}\left(\sum_{\xi \in \mathbf{T}^{*}}^{\xi \neq 0} \frac{\left|m_{\xi}\right|^{2}}{\|\xi\|^{d+1}} \Psi_{f}\left(u^{-1}\|\xi\|\right)\right) u^{d+1}= \\
=\frac{-\mathfrak{g}_{f}^{\prime}(0+)}{2 \pi^{2} \kappa_{d-1}}\left(\sum_{\xi \in \mathbf{T}^{*}}^{\xi \neq 0} \frac{\left|m_{\xi}\right|^{2}}{\|\xi\|^{d+1}}\right) \Phi_{f}\left(u^{-1}\right) u^{d+1}=
\end{gathered}
$$

and Eq. 5 yields

$$
=C_{\mu}\left(-\frac{d \kappa_{d}}{\kappa_{d-1}} \mathfrak{g}_{f}^{\prime}(0+)\right) \Phi_{f}\left(u^{-1}\right) u^{d+1} .
$$

It follows from the definition of derivative that

$$
\frac{-\mathfrak{g}_{f}^{\prime}(0+)}{2 \pi^{2} \kappa_{d-1}}=\frac{1}{2 \pi^{2} \kappa_{d-1}} \lim _{h \rightarrow 0+} \frac{1}{h}\left(\mathfrak{g}_{f}(0)-\mathfrak{g}_{f}(h)\right)=
$$

and by the use of Eq. 1 and 2 we have

$$
=\lim _{h \rightarrow 0+} \frac{1}{h} \int_{0}^{\infty} L(h \rho) \rho^{d-1} \mathscr{F} \mathfrak{g}_{f}(\rho) d \rho=
$$

and applying (the following) Theorem 2 to the function $\rho^{d-1} \mathscr{F} \mathfrak{g}_{f}(\rho)$ we obtain finally

$$
=\lim _{R \rightarrow+\infty} \frac{1}{R} \int_{0}^{R} \rho^{d+1} \mathscr{F} \mathfrak{g}_{f}(\rho) d \rho,
$$

hence $\lim _{x \rightarrow+\infty} \frac{1}{x} \int_{0}^{x} \Psi_{f}(y) d y=1$ and $0 \leq \Psi_{f}$. The proof of $\lim _{x \rightarrow+\infty} \frac{1}{x} \int_{0}^{x} \Phi_{f}(y) d y=1$ and $0 \leq \Phi_{f}$ is straightforward.

Let $\mathbf{M}^{1}(\mathbb{R})$ be the normed space of all continuous functions $f$ such that

$$
\sum_{i=-\infty}^{\infty} \max \{|f(x)|, i<x<i+1\}<\infty .
$$

Wiener's second Tauberian theorem (Wiener, 1933, Theorem 5) says: Let $\psi$ have uniformly bounded variation on unit intervals in $\mathbb{R}, N \in \mathbf{M}^{1}(\mathbb{R}), \mathscr{F} N(\tau) \neq$ 0 for each $\tau \in \mathbb{R}$ and

$$
\lim _{\eta \rightarrow \infty} \int_{-\infty}^{\infty} N(\eta-t) d \psi(t)=a \mathscr{F} N(0) .
$$

Then

$$
\lim _{\eta \rightarrow \infty} \int_{-\infty}^{\infty} f(\eta-t) d \psi(t)=a \mathscr{F} f(0)
$$

for every $f \in \mathbf{M}^{1}(\mathbb{R})$. 
Theorem 2. Let $f(r) \geq 0$ and $f \in \mathbf{L}_{\text {loc }}^{1}\left(\mathbb{R}^{+}\right)$. Then

$$
\lim _{R \rightarrow \infty} \frac{1}{R} \int_{0}^{R} r^{2} f(r) d r=\lim _{h \rightarrow 0+} \frac{1}{h} \int_{0}^{\infty} L(h r) f(r) d r,
$$

where

$$
L(u)=\pi^{-\frac{3}{2}} \Gamma\left(\frac{d+1}{2}\right)\left(\frac{1}{\Gamma\left(\frac{d}{2}\right)}-\frac{J_{\frac{d}{2}-1}(2 \pi u)}{(\pi u)^{\frac{d}{2}-1}}\right),
$$

whenever one of the limits exists.

Proof. We may set $f=0$ in $(0,1)$ according to the theorem on monotone convergence.

By the substitution $r=\exp (t)$ and defining

$$
\begin{gathered}
R=\exp (\eta)=\frac{1}{h}, \quad r^{2} f(r)=\varphi(t), \\
N_{1}(s)=I_{\{s \mid s>0\}} \exp (-s), \\
N_{2}(s)=\exp (s) L(\exp (-s)), \\
\psi(t)=\int_{0}^{t} \varphi(s) d s,
\end{gathered}
$$

we obtain an equivalent formulation of the theorem:

$$
\lim _{\eta \rightarrow \infty} \int_{-\infty}^{\infty} N_{1}(\eta-t) d \psi(t)=\lim _{\eta \rightarrow \infty} \int_{-\infty}^{\infty} N_{2}(\eta-t) d \psi(t) .
$$

whenever at least one of the two limits exists. We have $N_{i}(\eta)>0$ for $0 \leq \eta \leq 1$ and $i=1,2$, thus, if one of the above limits exists, then

$$
\limsup \int_{n}^{n+1} d \psi(s)<\infty
$$

and also

$$
\int_{n}^{n+1} d \psi(s)<M<\infty,
$$

i.e. the variation of $\psi$ on unit intervals is bounded by $M<\infty$. $N_{2}$ :

We will evaluate the Fourier transform of kernel

$$
\mathscr{F} N_{2}(\tau)=\int_{0}^{\infty} L(u) u^{2 \pi i \tau-2} d u .
$$

Using $\int t^{-v} J_{v+1}(t) d t=-t^{-v} J_{v}(t)$ we get

$=\frac{2}{\sqrt{\pi}} \Gamma\left(\frac{d+1}{2}\right) \int_{0}^{\infty} u^{2 \pi i \tau-2} \int_{0}^{u}(\pi s)^{1-\frac{d}{2}} J_{\frac{d}{2}}(2 \pi s) d s d u=$ and integration by parts gives

$$
=\frac{2 \Gamma\left(\frac{d+1}{2}\right)}{\sqrt{\pi}(1-2 \pi i \tau)}\left(-\left[u^{2 \pi i \tau-1} \int_{0}^{u} \frac{J_{\frac{d}{2}}(2 \pi s)}{(\pi s)^{\frac{d}{2}-1}} d s\right]_{0}^{\infty}+\right.
$$

$$
\left.+\pi^{1-2 \pi i \tau} \int_{0}^{\infty} \frac{J_{\frac{d}{2}}(2 \pi u)}{(\pi u)^{\frac{d}{2}-2 \pi i \tau}} d u\right) .
$$

The first term in the parentheses is zero, because of l'Hospital rule and asymptotics of Bessel function. From the formula

$$
\int_{0}^{\infty} t^{a} J_{v}(2 t) d t=\frac{1}{2} \Gamma\left(\frac{v+a+1}{2}\right) \Gamma\left(\frac{v-a+1}{2}\right)^{-1},
$$

valid if Re $a<\frac{1}{2}$, Re $a+v>-1$, it follows that

$$
\mathscr{F} N_{2}(\tau)=\frac{\pi^{-2 \pi i \tau-\frac{1}{2}}}{1-2 \pi i \tau} \frac{\Gamma\left(\frac{d+1}{2}\right) \Gamma\left(\frac{1}{2}+\pi i \tau\right)}{\Gamma\left(\frac{d+1}{2}-\pi i \tau\right)} .
$$

Now it is easy to see that $\mathscr{F} N_{2}(0)=1$ and $\mathscr{F} N_{2}(\tau) \neq$ 0 for $\tau \in \mathbb{R}$, because Gamma function has no roots and all its poles are nonpositive integers.

The function $N_{1}$ is not continuous (thus $N_{1} \notin$ $\mathbf{M}^{1}(\mathbb{R})$ ). We set

$$
N_{1, \varepsilon}(t)=\frac{1}{\varepsilon} \int_{t}^{t+\varepsilon} N_{1}(s) d s
$$

for $\varepsilon>0$. Then $N_{1, \varepsilon} \in \mathbf{M}^{1}(\mathbb{R})$,

$$
\mathscr{F} N_{1, \varepsilon}(\tau)=\frac{\exp (2 \pi i \tau \varepsilon)-1}{2 \pi i \tau \varepsilon} \frac{1}{1+2 \pi i \tau}
$$

has no real root and $\mathscr{F} N_{1, \varepsilon}(0)=1$. Thus the limits are equal for $N_{1, \varepsilon}$ and $N_{2}$ if one of the limits exists by second Wiener's Tauberian theorem.

It follows from the obvious inequalities

$$
\frac{1-\exp (-\varepsilon)}{\varepsilon} N_{1}(t) \leq N_{1, \varepsilon}(t) \leq \frac{\exp (\varepsilon)-1}{\varepsilon} N_{1}(t)
$$

that

$$
\frac{\varepsilon}{\exp (\varepsilon)-1} N_{1, \varepsilon}(t) \leq N_{1}(t) \leq \frac{\varepsilon}{1-\exp (-\varepsilon)} N_{1, \varepsilon}(t),
$$

and because $\psi$ is nondecreasing the limits in the statement of the theorem with $N_{1}$ and $N_{2}$ are equal, if one of the limits exists. 
Let $K \subset \mathbb{R}^{d}$ be a measurable set. The perimeter of a measurable set $K \subset \mathbb{R}^{d}$ is the variation of its characteristic function $I_{K}$ (Ambrosio, 2000, Definition 3.35). If $\operatorname{Per}(K)$ is finite then the gradient of the characteristic function $I_{K}$ is the vector Radon measure $D I_{K}$ in the sense of distributions (Ambrosio, 2000, Theorem 3.36). We have the polar decomposition $D I_{K}=\nabla_{I_{K}}\left|D I_{K}\right|$, where the vector function $\nabla_{I_{K}}$ is the inner normal and the positive measure $\left|D I_{K}\right|$ is the variation of $D I_{K}$ (Ambrosio, 2000, Corollary 1.29).

Let the reduced boundary $\partial^{*} K$ be the set of points where the normal to the set $K$ exists (Ambrosio, 2000, Definition 3.54). Then $\left|D I_{K}\right|=\mathscr{H}^{d-1} \mid \partial^{*} K$ (Ambrosio, 2000, Theorem 3.59), where $\mathscr{H}^{d-1}$ is the Hausdorff measure.

The covariogram of $f I_{K}$ is

$$
g_{f I_{K}}(x)=\int_{K \cap K-x} f(y+x) \overline{f(y)} d y
$$

and the corresponding isotropic covariogram is

$$
\mathfrak{g}_{f I_{K}}(\|x\|)=\int_{\mathbf{S O}_{d}} g_{M\left(f I_{K}\right)}(x) d p(M) .
$$

Theorem 3. Let $K \subset \mathbb{R}^{d}$ be a measurable set. If $\operatorname{Per}(K)<\infty$ and $f \in \mathbf{C}_{c}^{1}\left(\mathbb{R}^{d}\right)$, then

$$
\mathfrak{g}_{f I_{K}}^{\prime}(0+)=-\frac{\kappa_{d-1}}{d \kappa_{d}} \int_{\partial^{*} K}|f|^{2}(x) d \mathscr{H}^{d-1}(x) .
$$

Proof. Let $u \in S^{d-1}$ and $\varepsilon>0$, then

$$
\begin{gathered}
\frac{g_{f I_{K}}(-\varepsilon u)-2 g_{f I_{K}}(0)+g_{f I_{K}}(\varepsilon u)}{2 \varepsilon}= \\
=-\frac{1}{2 \varepsilon} \int_{K \cap K-\varepsilon u}|f(x)-f(x+\varepsilon u)|^{2} d x- \\
-\frac{1}{2 \varepsilon} \int_{K \backslash K-\varepsilon u}|f(x)|^{2} d x-\frac{1}{2 \varepsilon} \int_{K-\varepsilon u \backslash K}|f(x+\varepsilon u)|^{2} d x=
\end{gathered}
$$

$|f|^{2}$ is a Lipschitz continuous function, thus the first integral converges to 0 and the function in the last one can be shifted by $\varepsilon u$, so that

$$
=-\frac{1}{2 \varepsilon} \int_{K \backslash K-\varepsilon u}|f(x)|^{2} d x-\frac{1}{2 \varepsilon} \int_{K-\varepsilon u \backslash K}|f(x)|^{2} d x+o(\varepsilon) \text {. }
$$

We have

$$
\frac{1}{\varepsilon} \int_{K \backslash K-\varepsilon u}|f|^{2}(x) d x-\frac{1}{\varepsilon} \int_{K-\varepsilon u \backslash K}|f|^{2}(x) d x=
$$

$$
\begin{gathered}
=\frac{1}{\varepsilon} \int_{K}|f|^{2}(x)-|f|^{2}(x-\varepsilon u) d x= \\
=\frac{1}{\varepsilon} \int_{K} \sum_{i=1}^{d} u_{i} \frac{\partial}{\partial x_{i}} \int_{-\varepsilon}^{0}|f|^{2}(x+t u) d t d x= \\
=\int_{\mathbb{R}^{d}} \frac{1}{\varepsilon} \int_{-\varepsilon}^{0}|f|^{2}(x+t u) d t u \cdot \nabla_{I_{K}}(x) d\left|D I_{K}\right|(x)
\end{gathered}
$$

where the last equality follows from the GaussGreen theorem (Ambrosio, 2000, Theorem 3.36). The integral converges to

$$
\int_{\mathbb{R}^{d}}|f|^{2}(x) u \cdot \nabla_{I_{K}}(x) d\left|D I_{K}\right|(x)
$$

because $|f|^{2}$ is uniformly continuous. Further, the integrals in the Gauss-Green equality above provide us with two expressions for the measure $\varepsilon^{-1} \mathscr{L}^{d}\left|(K \backslash K-\varepsilon u)-\varepsilon^{-1} \mathscr{L}^{d}\right|(K-\varepsilon u \backslash K)$ where $\mathscr{L}^{d}$ is the Lebesgue measure. The variance of this measure has density equal to $\varepsilon^{-1}$ with respect to $\mathscr{L}^{d}$ on the union of the two disjoint sets that form the Hahn decomposition of the measure (Ambrosio, 2000, Exercise 1.1). Convolution of the $\varepsilon^{-1}$ multiple of the Hausdorff measure on the interval $\varepsilon^{-1} \mathscr{H}^{1} \mid\langle 0, \varepsilon u\rangle$ with either the positive variation or with the negative variation of the directional derivative $u \cdot \nabla_{I_{K}}\left|D I_{K}\right|$ form another decomposition of the measure. By the use of minimality of the Hahn decomposition and of $|f|^{2} \geq 0$ we obtain

$$
\begin{aligned}
& \frac{1}{\varepsilon} \int_{K \backslash K-\varepsilon u}|f|^{2}(x) d x+\frac{1}{\varepsilon} \int_{K-\varepsilon u \backslash K}|f|^{2}(x) d x \leq \\
\leq & \int_{\mathbb{R}^{d}} \frac{1}{\varepsilon} \int_{-\varepsilon}^{0}|f|^{2}(x+t u) d t\left|u \cdot \nabla_{I_{K}}(x)\right| d\left|D I_{K}\right|(x)
\end{aligned}
$$

and both expressions converge to

$$
\int_{\mathbb{R}^{d}}|f|^{2}(x)\left|u \cdot \nabla_{I_{K}}(x)\right| d\left|D I_{K}\right|(x),
$$

the second one because of the uniform continuity of $|f|^{2}$ and the first one because of the lower semicontinuity of the variation with respect to locally weak ${ }^{*}$ convergence (Ambrosio, 2000, Proposition 1.62).

Finally, by the use of the Hausdorff measure on the reduced boundary (Ambrosio, 2000, Theorem 3.59) we obtain the limit of the difference quotient

$$
-\frac{1}{2} \int_{\partial * K}|f|^{2}(y)\left|u \cdot \nabla_{I_{K}}(y)\right| d \mathscr{H}^{d-1}(y) .
$$


and the statement of the theorem follows by averaging the integral over $u \in S^{d-1}$ using the identity

$$
\frac{1}{d \kappa_{d}} \int_{S^{d-1}}|u \cdot v| d \mathscr{H}^{d-1}(u)=2 \kappa_{d-1}\|v\| .
$$

Theorem 4. Let $K \subset \mathbb{R}^{d}$ be a measurable set with $\operatorname{Per}(K)<\infty$. Then

i)

$$
\begin{gathered}
\int_{\mathbf{S O}_{d}} \alpha \int_{F_{\mathbf{T}}}\left|\mu^{u}(M K+x)-\lambda\right| K||^{2} d x d p(M)= \\
=C_{\mu} \operatorname{Per}(K) \Phi_{K}\left(u^{-1}\right) u^{d+1},
\end{gathered}
$$

where $|K|$ is volume of $K$,

ii) if $f \in \mathbf{C}_{c}^{1}\left(\mathbb{R}^{d}\right)$, then

$$
\begin{aligned}
& \int_{\mathbf{S O}_{d}} \alpha \int_{F_{\mathbf{T}}}\left|\widehat{M f I_{K}} \star \mu^{u}(x)-\lambda J_{f}\right|^{2} d x d p(M)= \\
= & C_{\mu}\left(\int_{\partial^{*} K}|f|^{2}(x) d \mathscr{H}^{d-1}(x)\right) \Phi_{f I_{K}}\left(u^{-1}\right) u^{d+1},
\end{aligned}
$$

iii) and if $f_{1}, f_{2} \in \mathbf{C}_{c}^{1}\left(\mathbb{R}^{d}\right)$, then

$$
\begin{gathered}
\int_{\mathbf{S O}_{d}} \alpha \int_{F_{\mathbf{T}}}\left(\widehat{M f_{1} I_{K}} \star \mu^{u}(x)-\lambda J_{f_{1}}\right) . \\
\widehat{\left(\widehat{M f_{2} I_{K}} \star \mu^{u}(x)-\lambda J_{f_{2}}\right)} d x d p(M)= \\
=C_{\mu}\left(\operatorname{Re} \int_{\partial^{*} K} f_{1}(x) \overline{f_{2}(x)} d \mathscr{H}^{d-1}(x)\right) . \\
\cdot \Phi_{f_{1} I_{K}, f_{2} I_{K}}\left(u^{-1}\right) u^{d+1},
\end{gathered}
$$

where $\Phi$. are nonnegative functions on $\mathbb{R}^{+}$such that $\lim _{x \rightarrow+\infty} \frac{1}{x} \int_{0}^{x} \Phi .(y) d y=1$.

Proof. i) follows from Theorem 1 and from the equation $-d \kappa_{d} \mathfrak{g}_{K}^{\prime}(0+)=\kappa_{d-1} \operatorname{Per}(K)$ (Galerne, 2011, Theorem 14).

ii) Theorem 1 with the value of the right derivative of the isotropic covariogram in 0 obtained in Theorem 3 proves the statement.

iii) The cross-covariogram of functions $f_{1} I_{K}$ and $f_{2} I_{K}$ is

$$
g_{f_{1} I_{K}, f_{2} I_{K}}(x)=\int_{K \cap K-x} f_{1}(y+x) \overline{f_{2}(y)} d y
$$

and the corresponding isotropic cross-covariogram is defined as

$$
\mathfrak{g}_{f_{1} I_{K}, f_{2} I_{K}}(\|x\|)=\int_{\mathbf{S O}_{d}} g_{M\left(f_{1} I_{K}\right), M\left(f_{2} I_{K}\right)}(x) d p(M) .
$$

Obviously,

$$
\mathfrak{g}_{f_{1} \pm f_{2} I_{K}}=\mathfrak{g}_{f_{1} I_{K}} \pm 2 \mathfrak{g}_{f_{1} I_{K}, f_{2} I_{K}}+\mathfrak{g}_{f_{2} I_{K}}
$$

From

$$
\begin{gathered}
\int_{\partial^{*} K}\left|f_{1}(x) \pm f_{2}(x)\right|^{2} d \mathscr{H}^{d-1}(x)= \\
=\int_{\partial^{*} K}\left|f_{1}\right|^{2}(x) d \mathscr{H}^{d-1}(x) \pm \\
\pm 2 \operatorname{Re} \int_{\partial^{*} K} f_{1}(x) \overline{f_{2}(x)} d \mathscr{H}^{d-1}(x)+ \\
+\int_{\partial^{*} K}\left|f_{2}\right|^{2}(x) d \mathscr{H}^{d-1}(x)
\end{gathered}
$$

and by the use of Theorem 3 we obtain

$$
\begin{gathered}
-d \kappa_{d} \mathfrak{g}_{f_{1} I_{K}, f_{2} I_{K}}(0+)= \\
=\kappa_{d-1} \operatorname{Re} \int_{\partial^{*} K} f_{1}(x) \overline{f_{2}(x)} d \mathscr{H}^{d-1}(x) .
\end{gathered}
$$

The statement then follows from ii) applied to $\left(f_{1} \pm f_{2}\right) I_{K}$.

\section{DISCUSSION}

We obtained $\Phi \geq 0$ and $\lim _{x \rightarrow+\infty} \frac{1}{x} \int_{0}^{x} \Phi(y) d y=1$ in Theorem 4, which enables us to obtain reasonable approximation of the variance replacing $\Phi$ by 1 . The resulting formula can be used for selecting the proper sampling density in optimization of the sampling design efficiency (Gundersen and Jensen, 1987). Theorem 4 can be also used in cases when $f$ is smooth and $K$ is a bounded set with a finite perimeter.

We were not able to prove, that $\Phi$ is bounded, i.e. that the error of the approximation is $O\left(u^{d+1}\right), u \rightarrow$ $0+$, for a general set with finite perimeter. This can be proved easilly, if the Hankel transform of the isotropic covariogram of the set or of the function restricted on the set is $O\left(\rho^{-d-1}\right), \rho \rightarrow+\infty$. The asymptotics then could be prooved more easilly using the first Wiener's Tauberian theorem (Janáček, 2008). Isotropic covariograms of bounded convex sets or of $\mathbf{C}^{1.5}$ sets have this property (Brandolini et al., 2003). 


\section{ACKNOWLEDGMENT}

This work was supported by Czech Science Foundation grant No. P302/12/1207, by MEYS (project LM2015062 Czech-BioImaging, project CZ.02.1.01/0.0/0.0/16_013/0001775 Modernization and support of research activities of the national infrastructure for biological and medical imaging Czech-BioImaging funded by $\mathrm{OP} \mathrm{RDE}$ ) and $\mathrm{MH}$ CR-DRO (Institute for Clinical and Experimental Medicine IKEM, IN00023001).

\section{REFERENCES}

Ambrosio L, Fusco N, Pallara D (2000). Functions of bounded variation and free discontinuity problems, Oxford University Press.

Bochner S, Chandrasekharan K (1949). Fourier transform, Princeton University Press.

Brandolini L, Hofmann S, Iosevich A (2003). Sharp rate of average decay of the Fourier transform of a bounded set, Geom Funct Anal 13:671-80.

Galerne B (2011). Computation of the perimeter of measurable sets via their covariogram. Applications to random sets. Image Anal Stereol 30:39-51.
Gundersen HJG, Jensen EB (1987). The efficiency of systematic sampling in stereology and its prediction. J Microsc 147 (3):229-63.

Hlawka E (1950). Über integrale auf konvexen körpern I. Monatsh für Math 54 1-36.

Janáček J (2006). Variance of periodic measure of bounded set with random position. Comment Math Univ Carolinae 47:473-82.

Janáček J (2008). An asymptotics of variance of the lattice points count. Czechoslovak Mathematical Journal, 58 (133),751-75.

Kendall DG (1948). On the number of lattice points inside the random oval. Q J Math Oxford Ser 19 (3): $1-26$.

Kieu K, Mora M (2004). Asymptotics for geometric spectral densities and stochastic approach of the lattice-point problem. Math Notae XLII:77-93.

Kieu K, Mora M (2006). Precision of stereological planar estimators. J Microsc 222 (3):201-11.

Matheron G (1965). Les variables régionalisées et leur estimations. Masson et Cie, Éditeurs.

Rudin W (1987). Real and complex analysis. 3rd ed., McGraw-Hill.

Wiener N (1933). The Fourier integral and certain of its applications. Dover Publications Inc. 